\title{
Free Volume Approximation and Equation of State for the fcc Phase of Polydisperse Hard Spheres
}

\author{
K.V. Tretiakov*, K.W. Wojciechowski ${ }^{* *}$ \\ Institute of Molecular Physics, Polish Academy of Sciences,, Smoluchowskiego 17/19, 60-179 Poznań, Poland \\ ${ }^{*}$ E-mail: kvt@ifmpan.poznan.pl ${ }^{* *}$ E-mail: kww@ifmpan.poznan.pl
}

Received: 24 November 2012; revised: 7 January 2013; accepted: 14 January 2013; published online: 21 March 2013

\begin{abstract}
Monte Carlo simulations of the fcc phase of polydisperse hard spheres near close packing are reported. An experimental equation of state $(\mathrm{EoS})$ is determined numerically in the $N p T$ ensemble with variable shape of the periodic box. The close packing volume extrapolated from the obtained data shows a good agreement with earlier experiments performed by other methods. A new theoretical EoS, based on the free volume approximation, is proposed. The modified EoS fits experimental data for polydisperse hard spheres better than the approximation used before.
\end{abstract}

Key words: colloids, equation of state, hard spheres, Monte Carlo simulations, polydispersity

\section{INTRODUCTION}

Hard spheres, interacting through a hard potential, being infinity when any particles overlap and zero without any overlap, constitute one of the simplest models of various physical systems, like dense gases, simple liquids, glasses, amorphous metals, etc. The model of monodisperse (equidiameter) hard spheres has been extensively studied for more than fifty years and its thermodynamic and structural properties are well established [1-13]. In particular, it is known that monodisperse hard spheres exhibit the melting transition $[1,2,5]$ and their thermodynamically stable solid phase corresponds to the face centered cubic (fcc) crystalline phase [8-11].

In some materials, like colloidal suspensions [14], the particles are not identical but exhibit some size distribution around the average size, called polydispersity. To model them, polydisperse hard spheres are used $[14,15]$. The influence of polydispersity on the phase behavior of hard spheres has been investigated by experiments [15-18], computer simulations [19-24], density functional theories [25, 26], and simplified analytical approaches [22, 27-32]. Thermodynamic properties [33], elastic properties [34], and structure of crystals of hard colloidal spheres $[24,35,36]$ have been investigated. Recently, it has been shown that also in the case of polydisperse hard spheres (like for monodisperse hard spheres), the fcc structure is more stable than any hexagonal close packing (hcp) structure [37].

In the present paper the fcc crystal of polydisperse hard spheres is considered. The aim of the investigations is twofold. First, the EoS of the studied system is simulated near close packing in order to determine the close packing volume (CPV). The $\mathrm{CPV}$ of polydisperse hard spheres in the fcc crystalline phase is determined as a function of the size polydispersity. Second, an extension of the EoS obtained within the free volume approximation (FVA) [3, 4] for monodisperse hard spheres is proposed for description of the EoS of polydisperse hard spheres. It should be added here that the FVA is a kind of a mean field theory in which the configurational partition function of the system is approximated by a product of configurational partition functions of individual spheres moving in cages formed by their neighbours frozen in their site (average) positions. The applicability of the proposed EoS to the studied system is verified and compared with the version of the EoS known in literature $[31,32]$.

The paper is organized as follows. In Sec. II simulation details are described. In Sec. III the results of the simulations 
are discussed. Finally, conclusions are presented in Sec. IV.

\section{PRELIMINARIES}

\section{1. Simulation details}

All simulations were done for the fcc crystal of polydisperse hard spheres in the $N p T$ ensemble. The initial configurations corresponded to sphere centres forming an ideal fcc lattice occupying a cubic box of side $4 n \times \sqrt{2} a_{0}\left(a_{0}\right.$ is the nearest-neighbor distance) with periodic boundary conditions. Typical simulations were done for systems of $N=256$ particles, i.e. for $n=4$. Typical lengths of the runs were equal $10^{7}$ trial steps per particle (cycles), after equilibration. Some test runs performed for larger $N$ did not reveal any systematic errors exceeding the simulation errors. Therefore, one can expect that the data obtained for $N=256$ should approximate the thermodynamic limit with reasonable accuracy.

Remark: The runs performed in this work are much longer than standard runs necessary to determine the EoS. The reason is that during these runs not only the EoS but also elastic properties of the system were determined. The latter are the subject of a separate work.

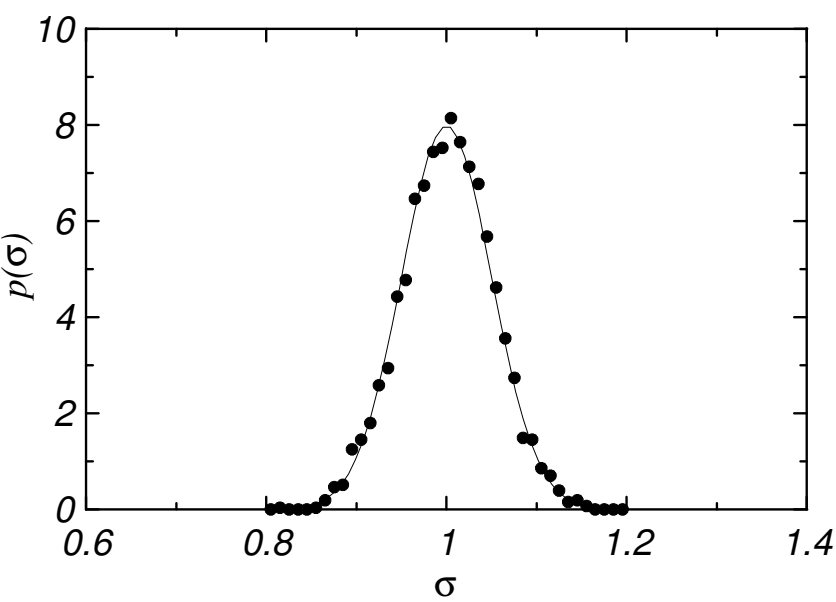

Fig. 1. Typical size distribution obtained for a system consisting of $N=256$ spheres with polydispersity $\delta=0.05 ; 10$ different initial structures were simulated

At the beginning of each simulation run the diameters $\sigma_{i}$ of the spheres centered on sites of an ideal fcc lattice were generated with a size distribution of a given polydispersity parameter, $\delta$. (In further discussion, the indices $i$ enumerating spheres are neglected. The initial lattice constant was chosen in such a way as to avoid overlaps of the spheres.) The polydispersity parameter was defined as the standard deviation of the particle size distribution, divided by the mean diameter of the particle $\langle\sigma\rangle$

$$
\delta=\frac{\sqrt{\left\langle\sigma^{2}\right\rangle-\langle\sigma\rangle^{2}}}{\langle\sigma\rangle} .
$$

The particle size distribution, $p(\sigma)$, was the Gaussian one $[20,22]$

$$
p(\sigma)=\frac{1}{\delta \sqrt{2 \pi}} \exp \left[\frac{-(\sigma-\langle\sigma\rangle)^{2}}{2 \delta^{2}}\right] .
$$

To obtain reasonable statistics, simulations of a given phase point were performed at least for 10 different initial structures (i.e. distributions of sphere diameters on the lattice sites) and then average values of volume and density were computed. The typical size distribution obtained during a simulation using the above mentioned procedure is shown in Fig. 1.

The terminus polydispersity, i.e. the polydispersity above which no fluid-solid coexistence and no crystallization can occur [38] for the hard polydisperse spheres, is about 0.07 . That is why the simulations discussed in this paper were performed within the range $0<\delta \leq 0.06$, where the solid phase of polydisperse spheres is stable and well defined.

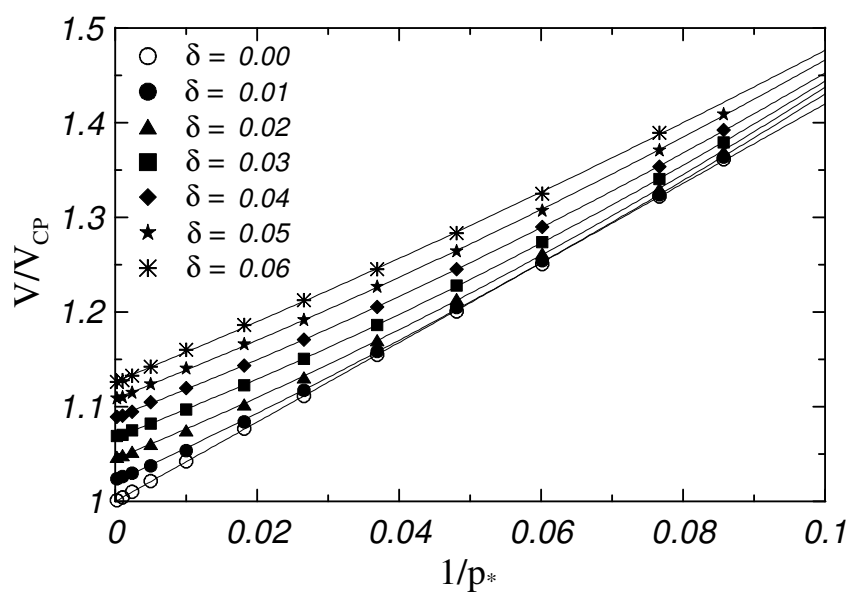

Fig. 2. The inverse pressure dependence of the relative volume for polydisperse hard sphere systems at a few polydispersities. The lines are polynomial fits to the data points

\section{2. Definitions and dimensionless quantities}

The volume corresponding to the most dense packing of hard bodies is called close packing.

The simulations were performed using the following dimensionless variables. The reduced volume of the hard sphere system is defined as $v^{*} \equiv V / V_{C P}$ where $V$ is the volume of the system and $V_{C P}=N \sigma^{3} / \sqrt{2}$ is the volume of $N$ monodisperse hard spheres of diameter $\sigma$ at close packing. The reduced volume of the polydisperse hard spheres is denoted by $v^{*}(\delta) \equiv V / V_{C P}^{\delta}$ where $V_{C P}^{\delta}$ is the volume of $N$ 
polydisperse hard spheres of the polydispersity parameter $\delta$ at close packing. The dimensionless pressure in polydisperse system is $p^{*} \equiv p\langle\sigma\rangle^{3} / k T$, where $k$ is the Boltzmann constant and $T$ is the temperature, and $\langle\sigma\rangle \equiv \sigma$ for the monodisperse system. The packing fraction is defined as $\phi=\pi\langle\sigma\rangle^{3} \mathrm{~N} / 6 \mathrm{~V}$ and its maximum value corresponds to the volume at close packing.

\section{RESULTS AND DISCUSSION}

\section{1. Close packing}

At any positive temperature $T$ the close packing can be reached only at infinite pressure $p$. Thus, the practical way of determinig the close packing volume is by taking the limit $1 / p \rightarrow 0$.

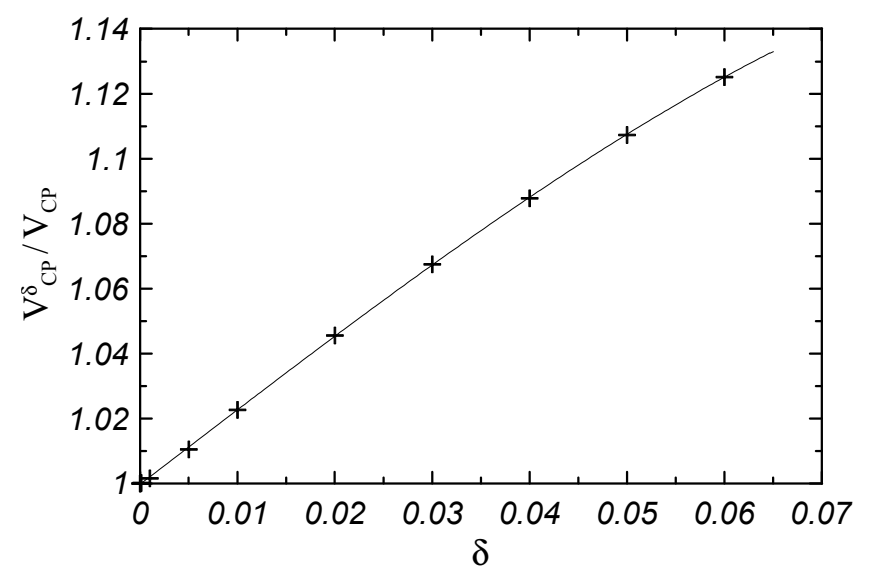

Fig. 3. The reduced close-packing volume of the polydisperse hard sphere system as a function of polydispersity. The line represents a fit to the data described by eq.(3)

In order to determine the close packing volume $V_{C P}^{\delta}$ for given polydispersity $\delta$ and to calculate the maximum packing fraction $\left(\phi_{\max }=\pi V_{C P} / 3 \sqrt{2} V_{C P}^{\delta}\right)$ as a function of polydispersity, one can extrapolate to zero the dependence of the relative volume of the studied system on the inverse pressure, see Fig. 2. It can be seen in Fig.2 that the close-packing volume grows with increasing polydispersity. The values of the close packing volumes $V_{C P}^{\delta}$ were determined from linear extrapolations done for 5 highest density points in Fig.2. The dependence of the reduced close packing volume on polydispersity is shown in Fig. 3. This dependence is well described by the following formula

$$
\frac{V_{C P}^{\delta}}{V_{C P}}=1+2.2989 \delta+0.34759 \delta^{2}-63.8898 \delta^{3} .
$$

In Fig. 4 the maximum packing fraction is presented as a function of polydispersity. To check the reliability of the simulations performed in this work, the obtained results are com- pared with the literature data [20]; a good agreement can be seen.

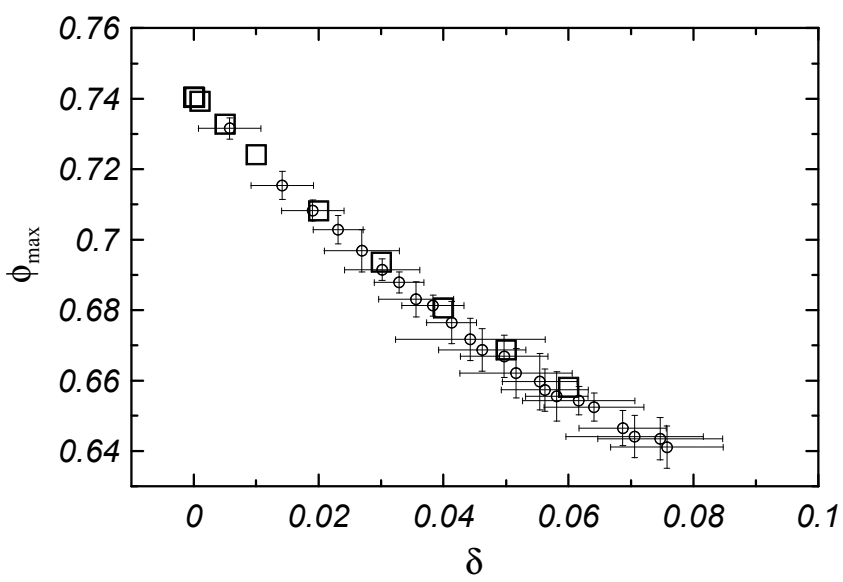

Fig. 4. Maximum packing fraction versus polydispersity for the fcc phase. Results of the present work are marked by open squares.

Small circles represent results of simulations done in Ref. [20]

\section{2. $\mathrm{EoS}$}

The EoS of hard sphere solids is well approximated by the free volume theory which predicts the following asymptotic dependence of the pressure, $p$, on the volume of the system $[3,4,13]$

$$
p_{F V}=\frac{a}{V / V_{C P}-1} \frac{k T}{\sigma^{3}}=\frac{a}{v^{*}-1} \frac{k T}{\sigma^{3}},
$$

where $a=3 \sqrt{2}$.

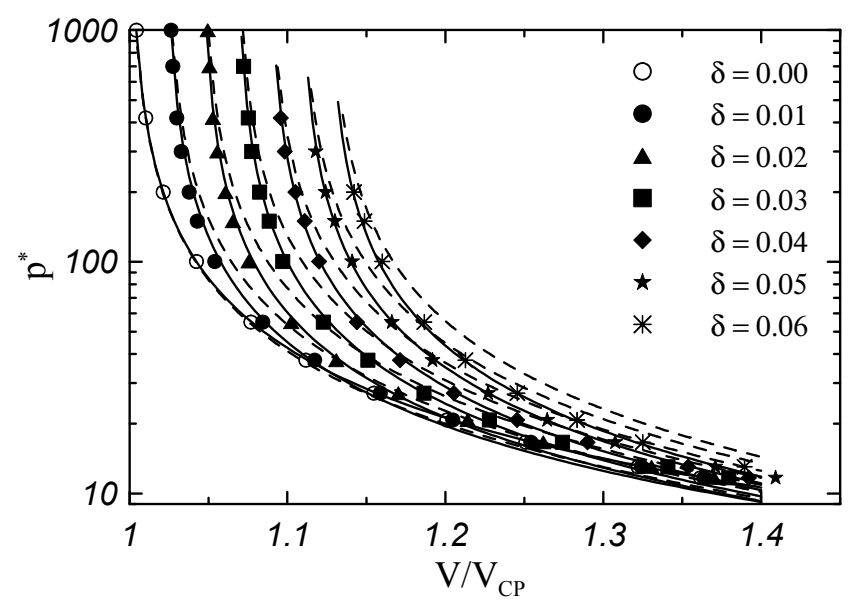

Fig. 5. The equation of state of polydisperse hard spheres. The dashed lines correspond to eq.(5). The solid lines correspond to eq.(6)

The free volume theory for polydisperse hard sphere system was proposed by $\mathrm{Xu}$ and Baus [31] and used by Huang and $\mathrm{Xu}[32]$ in a study of three-component mixtures of hard 
spheres. The EoS of the hard sphere solids obtained within this approximation was written $[31,32]$ in the form

$$
p_{F V}=\frac{\rho}{1-\left(\phi / \phi_{\max }\right)^{1 / 3}} \frac{k T}{\sigma^{3}},
$$

where $\rho=N / V$ is the number density. In Fig. 5, the results of the MC simulations performed in this work are compared with the theoretical approximation (5), see dashed lines. As expected, the agreement between the experiment and theory is good at high pressures.

It can be seen, however, in the same figure (Fig. 5) that at lower pressures the dashed lines show worse agreement with the MC data than continuous lines. The latter represent the equation below

$$
p^{*}=\frac{p_{F V}\langle\sigma\rangle^{3}}{k T}=\frac{a(\delta)}{V / V_{C P}^{\delta}-1}=\frac{a(\delta)}{v^{*}(\delta)-1},
$$

where $a(\delta)$ is presented in Fig. 6 and approximated by the equation $a(\delta)=2.7013+1.5353 /(1+124 \delta)$.

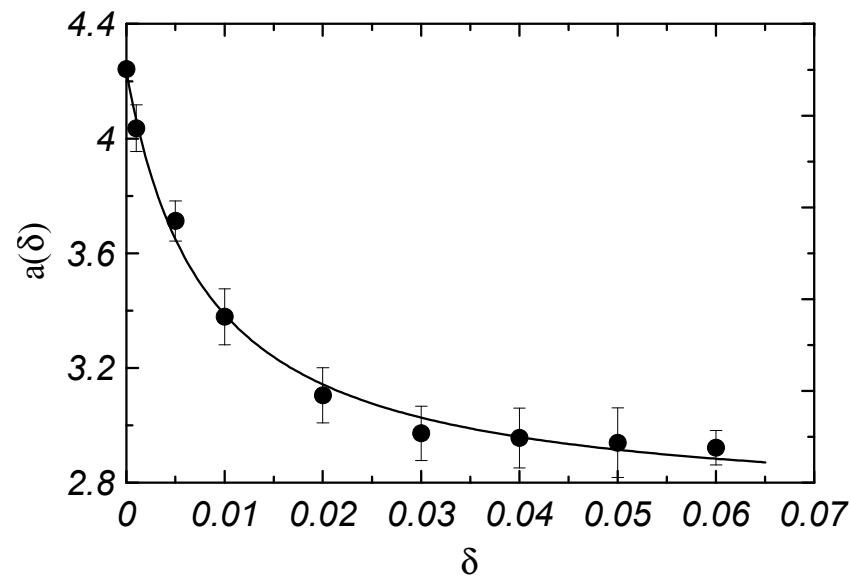

Fig. 6. Dependence of the coefficient $a(\delta)$ on the polydispersity. The line represents the fit to the data described in the text

Comparing (4) and (6), and taking into account the definition (3), one can see that (6) is obtained by replacing the close packing volume $V_{C P}$ of equidiameter hard spheres in the formula (4) by $V_{C P}^{\delta}$ for the studied polydispersity $\delta$ and making the coefficient $a$ a function of the polydispersity $a(\delta)$. It can be easily checked that for monodisperse hard spheres both (5) and (6) have the same asymptotics at the close packing limit but differ at lower densities. The data in Fig. 6 were extracted from fits to simulation results using eq. (6) for each polydispersity, see Fig. 7.

Remark: The EoS described by (6) is based on the results obtained for systems of $N=256$ particles. In the thermodynamic limit (i.e. when $N \rightarrow \infty$ ) the values of the close packing volume $V_{C P}^{\delta}$ of polydisperse spheres can be slightly different. However, this may affect only the values of $v^{*}(\delta)$ and coefficients $a(\delta)$ but not the form of the equation (6).

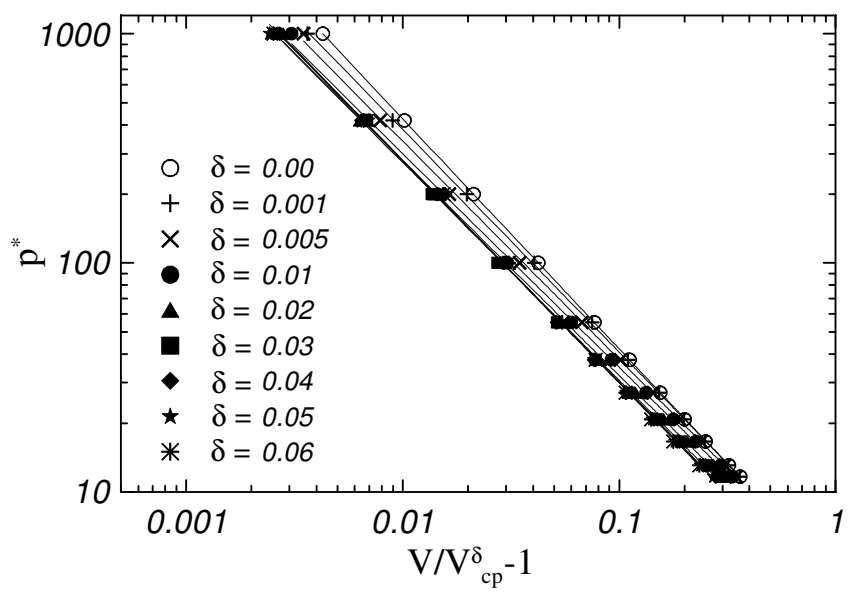

Fig. 7. Log-log plot of pressures of the polydisperse hard sphere system as functions of the 'excess' volume $V / V_{C P}^{\delta}-1$ for various $\delta$. The solid line corresponds to the equation of state given by eq. (6)

\section{SUMMARY AND CONCLUSIONS}

The equation of state of the fcc polydisperse hard sphere crystals was determined by Monte Carlo simulations in the $N p T$ ensemble with a variable shape of the periodic box. The obtained results are in good agreement with earlier literature data obtained by other methods [20]. The dependence of the close packing volume of polydisperse hard spheres on polydispersity was determined and approximated by a polynomial fit. The EoS, described by (6), has been proposed for polydisperse hard spheres, obtained by generalization of a variant of FVA for monodisperse hard spheres. The new EoS describes the simulation data better than the approximation (5) used earlier.

\section{Acknowledgments}

KVT thanks Professor Alexander Pankov (Gomel State Technical University) for useful discussions. This work was supported by grant NN202261438 of the Polish Ministry of Science and Education. Part of the calculations was performed at Poznań Computer and Networking Center (PCSS).

\section{References}

[1] W.W. Wood and J.D. Jacobson, Preliminary results from a recalculation of the Monte Carlo equation of state of hard spheres, J. Chem. Phys. 27, 1207 (1957).

[2] B.J. Alder and T.E. Wainwright, Phase transition for hard sphere system, J. Chem. Phys. 27, 1208 (1957). 
[3] F.H. Stillinger, R.L. Kornegay, and E.A. DiMarzio, Systematic approach to explanation of rigid disk phase transition, $\mathrm{J}$. Chem. Phys. 40, 1564 (1964).

[4] F.H. Stillinger and Z.W. Salsburg, Elasticity in rigid-disk and sphere crystals, J. Chem. Phys. 46, 3962 (1967).

[5] W.G. Hoover and F.H. Ree, Melting transition and communal entropy for hard spheres, J. Chem. Phys. 49, 3609 (1968).

[6] D. Frenkel and A.J. C. Ladd, Elastic constants of hard-sphere crystals, Phys. Rev. Lett. 59, 1169 (1987).

[7] K.J. Runge and G.V. Chester, Monte-Carlo determination of the elastic constants of the hard-sphere solid, Phys. Rev. A 36, 4852 (1987).

[8] L.V. Woodcock, Entropy difference between the face-centred cubic and hexagonal close-packed crystal structures, Nature 385, 141 (1997).

[9] P.G. Bolhuis, D. Frenkel, S.-Ch. Mau, and D.A. Huse, Entropy difference between crystal phases, Nature 388, 235 (1997).

[10] L.V. Woodcock, Entropy difference between crystal phases Reply, Nature 388, 236 (1997).

[11] K.V. Tretiakov and K.W. Wojciechowski, Efficient Monte Carlo simulations using a shuffled nested Weyl sequence random number generator, Phys. Rev. E 60, 7626-7628 (1999).

[12] O. Farago and Y. Kantor, Fluctuation formalism for elastic constants in hard-spheres-and-tethers systems, Phys. Rev. E 61, 2478 (2000).

[13] K.V. Tretiakov and K.W. Wojciechowski, Poisson's ratio of the fcc hard sphere crystal at high densities, J. Chem. Phys. 123, 074509 (2005).

[14] P. Salgi and R. Rajagopalan, Polydispersity in colloids - implications to static structure and scattering, Advances in Colloid and Interface Science 43, 169-288 (1993).

[15] P.N. Pusey and W. van Megen, Phase-behavior of concentrated suspensions of nearly hard colloidal spheres, Nature 320, 340 (1986).

[16] P.N. Pusey, Colloidal suspensions, in: J.P. Hansen, D. Levesque, and J. Zinn-Justin, editors, Liquids, Freezing and the Glass Transition Elsevier, 1991.

[17] M.E. Leunissen, Ch. G. Christova, A.-P. Hynninen, C.P. Royall, A.I. Campbell, M. Dijkstra A. Imhof, R. van Roij, and A. van Blaaderen, Ionic colloidal crystals of oppositely charged particles, Nature 437, 235 (2005).

[18] V.W.A. de Villeneuve, R.P.A. Dullens, D.G. A.L. Aarts, E. Groeneveld, J.H. Scherff, W.K. Kegel, and H.N.W. Lekkerkerker, Colloidal hard-sphere crystal growth frustrated by large spherical impurities, Science 309, 1231 (2005).

[19] E. Dickinson and R. Parker, Polydispersity and the fluidcrystalline phase-transition, J. Phys. Lett. 46, L229 (1985).
[20] P.G. Bolhuis and D.A. Kofke, Monte carlo study of freezing of polydisperse hard spheres, Phys. Rev. E 54, 634 (1996).

[21] P.G. Bolhuis and D.A. Kofke, Freezing of polydisperse hard spheres, Phys. Rev. E 59, 618 (1999).

[22] S.E. Phan, W.B. Russel, J.X. Zhu, and P.M. Chaikin, Effects of polydispersity on hard sphere crystals, J. Chem. Phys. 108, 9789 (1998).

[23] T. Nogawa, N. Ito, and H. Watanabe, Dynamical study of a polydisperse hard-sphere system, Phys. Rev. E 82, 021201 (2010).

[24] M. Yang and H. Ma, Solid-solid transition of the sizepolydisperse hard sphere system, J. Chem. Phys. 130, 031103 (2009).

[25] J.L. Barrat and J.P. Hansen, On the stability of polydisperse colloidal crystals, J. Phys. 47, 1547 (1986).

[26] R. McRae and A.D. J. Haymet, Freezing of polydisperse hard-spheres, J. Chem. Phys. 88, 1114 (1988).

[27] P.N. Pusey, The effect of polydispersity on the the crystallization of hard spherical colloids, J. Phys. 48, 709 (1987).

[28] R.P. Sear, Phase separation and crystallisation of polydisperse hard spheres, Europhys. Lett. 44, 531 (1998).

[29] P. Bartlett and P.B. Warren, Reentrant melting in polydispersed hard spheres, Phys. Rev. Lett. 82, 1979 (1999).

[30] P. Bartlett, Freezing in polydisperse colloidal suspensions, J. Phys.: Cond. Matt. 12, A275 (2000).

[31] H. Xu and M. Baus, Free-volume theory of the freezing of polydisperse hard-sphere mixtures: Initial preparation, fractionation, and terminal polydispersity, J. Chem. Phys. 118, 5045 (2003).

[32] C.C. Huang and H. Xu, Polydisperse hard sphere mixtures: equations of state and the melting transition, Mol. Phys. 102, 967 (2004).

[33] P. Bartlett, Thermodynamic properties of polydisperse hard spheres, Mol. Phys. 97, 685 (1999).

[34] M. Yang and H. Ma, Elasticity of a polydisperse hard-sphere csrystal, Phys. Rev. E 78, 011404 (2008).

[35] P.N. Pusey, W. van Megen, P. Barlett, B.J. Ackerson, J.G. Rarity, and S.M. Underwood, Structure of crystals of hard colloidal spheres, Phys. Rev. Lett. 63, 2753 (1989).

[36] J. Mattsson, H.M. Wyss, A. Fernandez-Nieves, K. Miyazaki, Zh. Hu, D.R. Reichman, and D.A. Weitz, Soft colloids make strong glasses, Nature 462, 83 (2009).

[37] M. Yang and H. Ma, Effect of polydispersity on the relative stability of hard-sphere crystals, J. Chem. Phys. 128, 134510 (2008).

[38] M. Fasolo and P. Sollich, Equilibrium phase behavior of polydisperse hard spheres, Phys. Rev. Lett. 91, 068301 (2003). 


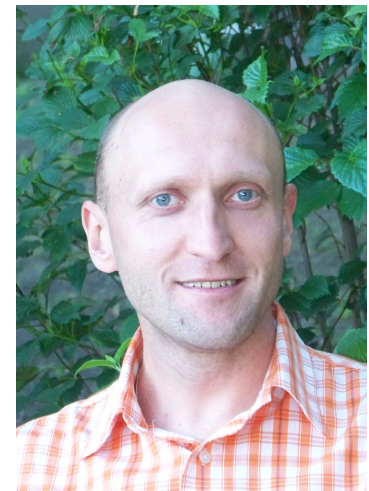

Konstantin V. Tretiakov graduated summa cum laude in industrial electronics from the The Pavel Sukhoi State Technical University (Gomel, Belarus) in 1994. He received his PhD degree in physics from the Institute of Molecular Physics, Polish Academy of Sciences (Poznan, Poland) in 2000. In 2002-2004, Dr. Tretiakov was a Postdoctoral Fellow (with Prof. S. Scandolo) in the Condensed Matter and Statististical Physics Section at the Abdus Salam International Center for Theoretical Physics (Trieste, Italy). In 2007-2009, Dr. Tretiakov worked with Prof. B. Grzybowski in the Department of Chemical and Biological Engineering at Northwestern University (Evanston IL, USA). He is a staff member of Prof. K.W. Wojciechowski group at the Institute of Molecular Physics. He specializes in statistical physics and computer simulation of many body systems, with research focusing on non-equilibrium self-assembling systems, transport properties of soft-matter, and elastic properties of solids, in particular materials exhibiting negative Poisson's ratio.

Krzysztof W. Wojciechowski is the Full Professor and the Head of the Division of Soft Matter Physics and Functional Materials and the Group of Computational Physics of Complex Systems at the Institute of Molecular Physics of the Polish Academy of Sciences. He received MSc degree in physics and MSc degree in mathematics from the Adam Mickiewicz University in Poznan. He earned the PhD in physics from the Institute of Molecular Physics, where he also habilitated. His research interests concern, amongst other topics, statisticalmechanical properties of hard-body systems (statistical-mechanical geometry), algorithms for simulations of many-body systems, auxetics and influence of various mechanisms on the Poissons ratio of condensed matter systems, materials with unstable inclusions, generators of (pseudo)random numbers, applications of fractional derivative in physics, and exotic liquid crystalline phases. For more details, see: http: //www.ifmpan.poznan.pl/zp10/zp10_www.htm. 